\title{
Photography in Dentistry: \\ Theory and Techniques in Modern Documentation
}

\section{Pasquale Loiacono, Luca Pascoletti}

Quintessence Publishing Company, Inc

Language: English

336 pages, 847 color illustrations

ISBN: 978-88-7492-169-0

Publication Date: February, 2012

Price: $122.00 €$

Nowadays, when dentistry resorts more and more to the concept of evidence-based medicine, the dentist uses images to communicate with the patient or with the dental technician, in order to record the clinical situation in the pre-treatment phase, for medical or forensic considerations or for a scientific presentation. As dental photography does not yet have a set of standards allowing reproducibility for clinical and scientific documentation, the authors present, in 13 chapters, the guidelines for modern photographic documentation.

The book is structured in two parts: "Theory" and "Techniques".

In the first part, "Theory", divided in nine chapters, the general principles, the optical system, the exposure concepts, the principles of digital photography, the role of photography in clinical practice, the settings of the camera for dentistry, the orthography of images, the flash units and the photographing radiographs are presented.

The second part, "Techniques", is divided in four chapters. The authors eloquently describe the equipment and accessories, and the extraoral and intraoral settings.

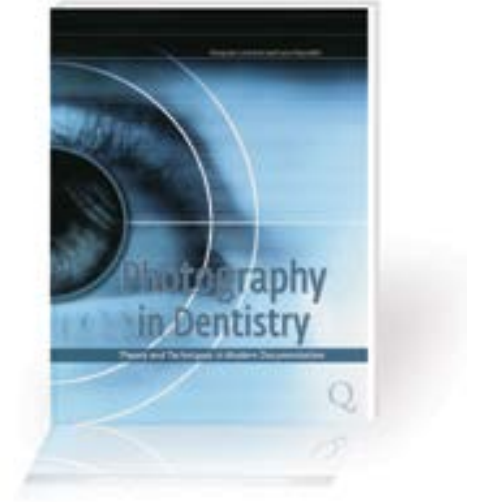

In the chapter "Equipment and Accessories", cameras and accessories (intraoral mirrors, cheek retractors and additional accessories), image quality and synergy between practitioner and assistant are presented.

The following chapters describes "Extraoral Series" and "Intraoral Series" suggestively illustrating all the norms and positions which must be known for an eloquent photographic documentation.

The last chapter includes documentary photography for orthodontics, periodontics, prosthetics, conservative dentistry and the communication with the dental technician.

In its 336 pages, the book contains 847 highquality illustrations, being a necessary guide for any dentist who aims to succeed in dental photography. 